\title{
DNA Damage Repair (DDR) Mutations and the Utility of High-Risk Genetics Clinics in Metastatic Castration- Refractory Prostate Cancer (mCRPC)
}

\author{
Nityam Rathi ${ }^{a}$, Neysi Anderson ${ }^{\mathrm{a}}$, Samantha Greenberg ${ }^{\mathrm{a}}$, Jennie Vaghera, \\ Neeraj Agarwal ${ }^{\mathrm{a}, \mathrm{b}}$, Andrew W. Hahn ${ }^{\mathrm{a}, \mathrm{b}}$
}

\begin{abstract}
Germline pathogenic variants (PVs) in DNA-repair genes have garnered increasing attention in metastatic prostate cancer, and more patients are having somatic and germline DNA testing performed. Interpretation of germline DNA testing is a novel challenge for many clinicians, and the results of germline DNA-repair gene testing have significant implications for men with advanced prostate cancer and their children and siblings. Here, we report the case of a man with metastatic castration-refractory prostate cancer and a pathogenic, germline $B R C A 2$ variant. We discuss the significance of his referral to a high-risk genetics clinic and the unique targeted therapy that he responded to.
\end{abstract}

Keywords: Germline pathogenic variants; DNA damage repair genes; DNA damage repair mutations; PARP inhibitors; High-risk genetics clinic; Hereditary clinic; Metastatic castration-refractory prostate cancer

\section{Introduction}

Prostate cancer accounts for one in every five cancer diagnoses, making it the most common cancer in men [1]. The natural history of prostate cancer varies widely from an indolent course where patients typically have minimal symptomatic burden to more aggressive metastatic disease that is generally incurable. After changes to the United States Preventative Services Task Force (USPSTF) guidelines, the incidence of metastatic prostate cancer may have increased $[2,3]$. Due to its incurable na-

Manuscript submitted August 14, 2018, accepted August 27, 2018

${ }^{a}$ Division of Oncology, Department of Internal Medicine, Huntsman Cancer Institute, University of Utah, Salt Lake City, UT, USA

${ }^{b}$ Corresponding Author: Neeraj Agarwal, Department of Internal Medicine, Huntsman Cancer Institute, University of Utah, 2000 Circle of Hope, Ste. 2123, Salt Lake City, UT 84112, USA. Email: neeraj.agarwal@hci.utah.edu; Andrew W. Hahn, Department of Internal Medicine, Huntsman Cancer Institute, University of Utah, 2000 Circle of Hope, Ste. 2123, Salt Lake City, UT 84112, USA. Email: andrew.hahn@hsc.utah.edu

doi: https://doi.org/10.14740/wjon1144w ture, most men diagnosed with metastatic hormone-sensitive prostate cancer (mHSPC) will eventually progress to metastatic castration-resistant prostate cancer (mCRPC). While there are currently six treatments approved for $\mathrm{mCRPC}$, the diagnosis still carries a poor prognosis with median survival between 24 and 36 months [4]. Predictive biomarkers are not routinely used to optimize treatment selection among the agents available for mCRPC.

Recently, germline pathogenic variants (PVs) in DNArepair genes have garnered increasing attention in metastatic prostate cancer. A seminal paper by Pritchard and colleagues found that inherited or germline DNA-repair gene variants are present in $11.8 \%$ of men with metastatic prostate cancer [5]. Furthermore, in a phase 2 clinical trial, men with mCRPC and deleterious mutations in DNA-repair genes were shown to have high response rates to olaparib, a poly(adenosine diphosphate (ADP)-ribose) polymerase (PARP) inhibitor [6]. Due to the high prevalence of inherited or germline DNA-repair gene mutations in metastatic prostate cancer, and potential implications of these findings on family members, NCCN guidelines now recommend germline DNA testing in all men with metastatic prostate cancer and patients with $B R C A 1 / 2 \mathrm{PVs}$ found in somatic tumor testing [7]. However, interpretation of germline DNA sequencing is a novel and daunting challenge for many practicing oncologists.

Here, we report a case of mCRPC that demonstrates the utility of multi-disciplinary, high-risk genetics clinics and is the first report on the efficacy of a PARP inhibitor in a man with mCRPC with germline DNA-repair gene PVs outside of clinical trial.

\section{Case Report}

In December 2014, a 67-year-old male with a past medical history of coronary artery disease, ischemic cardiomyopathy, diabetes mellitus, hypertension, hyperlipidemia and hypothyroidism was found to have a prostate-specific antigen (PSA) of $6.6 \mathrm{ng} / \mathrm{mL}$ on a routine screening PSA. On digital rectal exam, a large right-sided, hard nodule extending from the apex and mid-gland near the base was noted. Subsequently, a prostatic biopsy demonstrated adenocarcinoma of the prostate with a Gleason score of $8(4+4)$. Computed tomography (CT) abdomen/pelvis and a bone scan showed no evidence of extra- 
prostatic disease.

A laparoscopic radical prostatectomy demonstrated adenocarcinoma of the prostate (Gleason 4+4; pT2c Nx Mx) with positive apical margins bilaterally and perineural invasion. Post-prostatectomy PSA was $6.9 \mathrm{ng} / \mathrm{mL}$. Given the positive surgical margins and elevated post-prostatectomy PSA, the patient was referred to radiation oncology where definitive radiotherapy was administered. Within 1 month of radiation, a restaging $\mathrm{CT}$ abdomen/pelvis showed pelvic and external iliac lymphadenopathy consistent with metastatic disease. Bone scans remained negative for bony metastases, so the patient was started on leuprolide for his new metastatic hormone-sensitive prostate cancer (mHSPC).

One year later in August 2016, the patient experienced PSA progression and was diagnosed with metastatic castration-resistant prostate cancer (mCRPC). For his newly diagnosed mCRPC, he was treated with eight cycles of first-line docetaxel. After eight cycles, his PSA was undetectable and he had near resolution of his metastatic disease. However, in July 2017, he had radiographic and PSA progression. Tumor tissue next-generation sequencing (NGS) (FoundationOne) sent from his initial prostatectomy revealed two frameshift variants in BRCA2 (N986fs*2 and V1283fs*2). The patient was then referred to Huntsman Cancer Institute's (HCI) Family Cancer Assessment clinic (FCAC), a high-risk genetics clinic. Given the high rate of concordance between somatic and germline BRCA1/2 PVs and NCCN recommendations to test all men with metastatic prostate cancer, genetic counselors recommended germline DNA testing [8]. Testing confirmed the presence of a germline $B R C A 2$ variant, aligned with his V1283fs*2 variant identified somatically. Given the patient's multiple cardiovascular co-morbidities and patient preference, treatment with next-generation androgen axis inhibitors was not pursued. Based on his germline and tumor PV in BRCA2 and efficacy data of olaparib from the above mentioned phase 2 trial, he was started on olaparib orally at $400 \mathrm{mg}$ BID at off trial in August 2017. Treatment with olaparib was well tolerated overall except for anemia that required a dose reduction to $300 \mathrm{mg}$ BID and eventually $200 \mathrm{mg}$ BID after 6 months of therapy. After 10 months of treatment, olaparib was discontinued due to radiographic and PSA progression.

Due to the presence of the patient's $B R C A 2$ variant, it was recommended that all of his first-degree relatives (FDRs) consider genetic testing for the specific mutation. Each of his FDRs had a $50 \%$ risk to also have this $B R C A 2$ mutation. All three of his children, and two of his siblings, were seen at the HCI's FCAC and found to have the familial BRCA2 variant. After discussion with genetic counselors, his two daughters elected to undergo prophylactic, bilateral mastectomy and oophorectomy, and his interested male relatives are followed by HCI's high-risk prostate cancer clinic.

\section{Discussion}

Genetic testing is becoming a common means of identifying both somatic and germline genetic abnormalities in cancer patients. In a 2016 multi-centered study, deleterious germline
DNA-repair gene mutations were observed in $11.8 \%$ of men with metastatic prostate cancer [5]. Herein, we present the case of a 68-year-old man with mCRPC who was initially found to have a $B R C A 2 \mathrm{PV}$ on tumor tissue NGS and confirmed on germline testing through our FCAC at HCI. After progressing on docetaxel, he responded to olaparib for approximately 10 months, which to our knowledge, is the first reported case on the efficacy of PARP inhibitor treatment for a germline DNArepair gene mutation outside of clinical trials. Furthermore, this case clearly demonstrates the utility of referral to multidisciplinary, high-risk genetics clinics for men with metastatic prostate cancer and mutations in DNA-repair genes identified on tumor sequencing. Our patient's referral led to the detection of germline BRCA variants in multiple relatives, resulting in preventative screening and prophylactic measures for family members.

The current NCCN guidelines for prostate cancer suggest germline DNA testing with all men who have advanced prostate cancer [7]. However, interpreting the results of germline DNA testing presents a novel challenge for many clinicians because mutations have differing pathogenicity, penetrance and clinical implications [9]. Furthermore, there are other genes outside of DNA-repair gene mutations that may influence outcomes in metastatic prostate cancer $[10,11]$. Thus, high-risk genetics clinics have the potential to improve outcomes for men with metastatic prostate cancer because genetic counselors can discuss the implications of germline testing and provide preventative measures to the family members of these men. Germline BRCA 1/2 mutations are a well-known risk factor for breast and ovarian cancer, among others, so as seen in our case, appropriate counseling can lead to important discussions with family members about prevention and prophylaxis, such as risk-reducing surgeries, screenings and medications.

The value of DNA-repair gene mutations as a predictive or prognostic biomarker for men with metastatic prostate cancer is still unclear at this time. In 2015, TOPARP-A, a multi-center, phase 2 clinical trial, demonstrated that men with mCRPC and homozygous deletions and/or deleterious mutations in DNA-repair genes have improved progression-free survival with olaparib, a PARP inhibitor, compared to men without DNA-repair genes (9.8 months versus 2.7 months) [6]. These findings suggest that DNA-repair mutations are a predictive biomarker of response to PARP inhibitors in metastatic prostate cancer. There are now many ongoing clinical trials evaluating PARP inhibitors alone or in combination with other treatments for men with mCRPC (Table 1). It is currently unclear whether DNA-repair gene mutations are predictive of response to androgen axis inhibitors, abiraterone and enzalutamide. In the past year, three retrospective cohort studies of similar size have evaluated whether DNA-repair gene mutations are predictive of response to androgen axis inhibitors [12-14]. All three studies have produced different, conflicting results, so the final verdict is still undecided.

In conclusion, this report describes the case of a man with mCRPC and germline BRCA2 variants who had a clinically meaningful and durable response to treatment with a PARP inhibitor, olaparib, outside of a clinical trial. Furthermore, his case clearly demonstrates the value of collaborating and referring to high-risk genetics clinics for germline testing as they 
Table 1. Completed and Ongoing Phase 2/3 Clinical Trials Evaluating PARP Inhibitors in Men With Metastatic Castration-Refractory Prostate Cancer

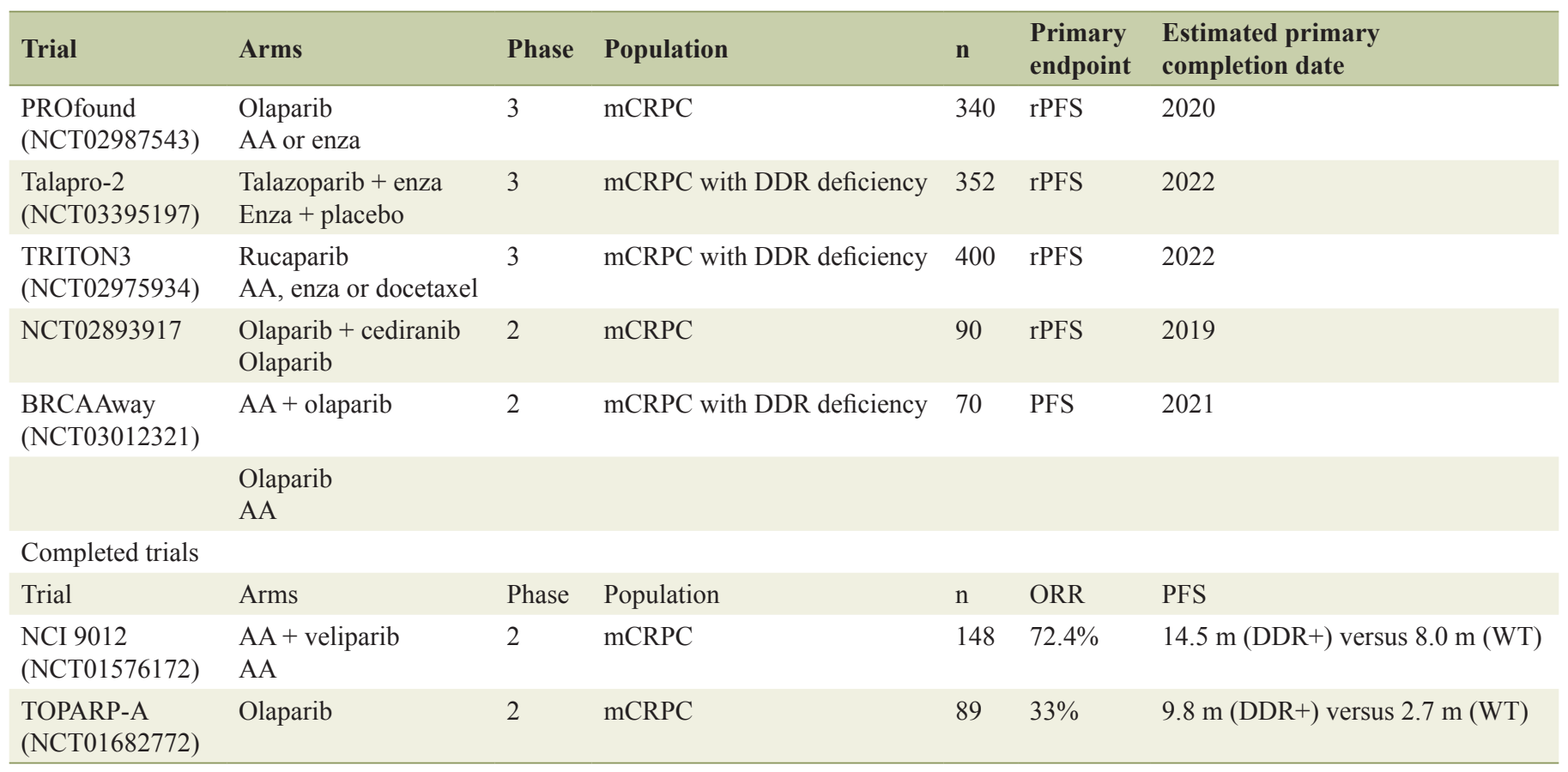

AA: abiraterone acetate plus prednisone; enza: enzalutamide; $\mathrm{mCRPC}$ : metastatic castration-refractory prostate cancer; rPFS: radiographic progression-free survival; DDR: DNA damage repair; ORR: objective response rate; PSA: prostate-specific antigen; PFS: progression-free survival. Primary completion date: the date on which the last participant in the clinical study was examined or received an intervention to collect final data for the primary outcome measure.

can impact treatment recommendations for men with DNArepair gene variants and their families.

\section{Conflict of Interest}

Nityam Rathi, Neysi Anderson and Andrew W. Hahn have no conflicts of interest to disclose. Neeraj Agarwal reports consultancy to Pfizer, Novartis, Merck, Genentech, Eisai, Exelixis, Clovis, EMD Serono, BMS, Astra Zeneca, Astellas and Ely Lilly. Jennie Vagher reports consultancy to Invitae Genetics. Samantha Greenberg reports consultancy to Tempus Laboratories.

\section{Financial Support}

No funding supported the preparation of this case report.

\section{References}

1. Siegel RL, Miller KD, Jemal A. Cancer statistics, 2018. CA Cancer J Clin. 2018;68(1):7-30.

2. Weiner AB, Matulewicz RS, Eggener SE, Schaeffer EM. Increasing incidence of metastatic prostate cancer in the United States (2004-2013). Prostate Cancer Prostatic Dis. 2016;19(4):395-397.

3. Dalela D, Sun M, Diaz M, Karabon P, Seisen T, Trinh QD,
Menon M, et al. Contemporary Trends in the Incidence of Metastatic Prostate Cancer Among US Men: Results from Nationwide Analyses. Eur Urol Focus. 2017.

4. Taplin ME. Drug insight: role of the androgen receptor in the development and progression of prostate cancer. Nat Clin Pract Oncol. 2007;4(4):236-244.

5. Pritchard CC, Mateo J, Walsh MF, De Sarkar N, Abida W, Beltran H, Garofalo A, et al. Inherited DNA-repair gene mutations in men with metastatic prostate cancer. N Engl J Med. 2016;375(5):443-453.

6. Mateo J, Carreira S, Sandhu S, Miranda S, Mossop H, Perez-Lopez R, Nava Rodrigues D, et al. DNA-repair defects and olaparib in metastatic prostate cancer. N Engl J Med. 2015;373(18):1697-1708.

7. Mohler JL, Armstrong AJ, Bahnson RR, D'Amico AV, Davis BJ, Eastham JA, Enke CA, et al. Prostate cancer, Version 1.2016. J Natl Compr Canc Netw. 2016;14(1):1930 .

8. Meric-Bernstam F, Brusco L, Daniels M, Wathoo C, Bailey AM, Strong L, Shaw K, et al. Incidental germline variants in 1000 advanced cancers on a prospective somatic genomic profiling protocol. Ann Oncol. 2016;27(5):795800.

9. Giri VN, Knudsen KE, Kelly WK, Abida W, Andriole GL, Bangma $\mathrm{CH}$, Bekelman JE, et al. Role of genetic testing for inherited prostate cancer risk: Philadelphia prostate cancer consensus conference 2017. J Clin Oncol. 2018;36(4):414-424. 
10. Eeles R, Goh C, Castro E, Bancroft E, Guy M, Al Olama AA, Easton D, et al. The genetic epidemiology of prostate cancer and its clinical implications. Nat Rev Urol. 2014;11(1):18-31.

11. Ewing CM, Ray AM, Lange EM, Zuhlke KA, Robbins $\mathrm{CM}$, Tembe WD, Wiley KE, et al. Germline mutations in HOXB13 and prostate-cancer risk. N Engl J Med. 2012;366(2):141-149.

12. Annala M, Struss WJ, Warner EW, Beja K, Vandekerkhove G, Wong A, Khalaf D, et al. Treatment outcomes and tumor loss of heterozygosity in germline DNA repair- deficient prostate cancer. Eur Urol. 2017;72(1):34-42.

13. Antonarakis ES, Lu C, Luber B, Liang C, Wang H, Chen Y, Silberstein JL, et al. Germline DNA-repair gene mutations and outcomes in men with metastatic castrationresistant prostate cancer receiving first-line abiraterone and enzalutamide. Eur Urol. 2018;74(2):218-225.

14. Mateo J, Cheng $\mathrm{HH}$, Beltran $\mathrm{H}$, Dolling $\mathrm{D}, \mathrm{Xu} \mathrm{W}$, Pritchard CC, Mossop H, et al. Clinical outcome of prostate cancer patients with germline DNA repair mutations: retrospective analysis from an international study. Eur Urol. 2018;73(5):687-693. 